\title{
Effect of Synoptic Systems on the Variability of the North Atlantic Oscillation
}

\author{
ULRIKE LÖPTIEN AND EBERHARD RUPRECHT \\ Leibniz-Institute for Marine Research, Kiel, Germany
}

(Manuscript received 13 July 2004, in final form 8 March 2005)

\begin{abstract}
The North Atlantic Oscillation (NAO) represents the dominant mode of atmospheric variability in the North Atlantic region. In the present study, the role of the synoptic systems (cyclones and anticyclones) in generating the NAO pattern is investigated. To study the intermonthly variations of the NAO, NCEPNCAR reanalysis data are used, and for the interdecadal variations the results of a 300-yr control integration under present-day conditions of the coupled model ECHAM4/OPYC3 are analyzed. A filtering method is developed for the sea level pressure anomalies. Application of this method to each grid point yields the low-frequency variability in the sea level pressure field that is due to the synoptic systems. The low-frequency variability of the filtered and the original data are in high agreement. This indicates that the low-frequency pressure variability, and with it the variability of the NAO, is essentially caused by the distribution of the synoptic systems. The idea that the distribution of the synoptic systems is the cause of the variation of the NAO is confirmed by high correlation between the latitudinal position of the polar front over the North Atlantic and the NAO index. Since most of the low-frequency variability in sea level pressure can be explained through the distribution of the synoptic systems, the NAO seems to be a reflection of the distribution of the synoptic systems, rather than the source for variations in the cyclone tracks.
\end{abstract}

\section{Introduction}

The North Atlantic Oscillation (NAO) is the dominant mode of the atmospheric circulation over the North Atlantic. It is generally represented by a northsouth dipole in the pressure or geopotential height field with centers near Greenland and over the central Atlantic, west of the Azores. This pattern is most pronounced during winter, and it explains a large fraction of the atmospheric variability in the North Atlantic region. It was first identified by Walker (1924) using correlation methods. The special attention that nowadays is paid to the NAO is due to its large influence on weather and climate, particularly over Europe. The most common index of the strength of the NAO is the difference between normalized monthly mean sea level pressure anomalies near the Azores and Iceland (Hurrell 1995; Jones et al. 1997). Other essentially equiva-

Corresponding author address: Dr. Ulrike Löptien, Ocean Circulation and Climate Dynamics, Leibniz-Institut für Meereswissenschaften an der Universität Kiel, Düsternbrooker Weg 20, D-24105 Kiel, Germany.

E-mail: uloeptien@ifm-geomar.de lent indices of the NAO have also been constructed (e.g., by an EOF analysis of the sea level pressure and geopotential height fields, where the time-dependent principal component gives the amplitude of the NAO index [Thompson and Wallace $(1998,2000)$; see also the review of Wanner et al. (2001)]. As shown by Wanner et al. (2001) with monthly and seasonal averages, the correlation between the various differently constructed NAO indices is high-at least during the winter season.

Time scales play an important role in connection with the NAO. The correlation increases between the two dipole centers with increasing time scale (Blackmon et al. 1984). The correlation coefficient between the sea level pressure anomalies near Iceland and the Azores during winter is -0.33 based on daily values and -0.56 for the 30-day running means [ $52 \mathrm{yr}$ of National Centers for Environmental Prediction-National Center for Atmospheric Research (NCEP-NCAR) reanalysis data]. Therefore the NAO index is mostly given as a monthly or seasonal mean.

Processes of different time scales are also involved in the investigation of the relationship between the NAO and the synoptic systems over the North Atlantic. 
Benedict et al. (2004) investigated individual NAO events and found that they originate from synopticscale waves. Numerous studies have been carried out to explain the relation between the NAO (defined as a monthly or seasonal mean) and storm tracks (Cai and Van den Dool 1991; Lau 1988; Ruprecht et al. 2002). They show that a close relation exists between the lowfrequency variability of the NAO and the highfrequency variations in sea level pressure or geopotential height fields. The meridional position of the storm tracks depends on the strength of the NAO. Usually two methods are applied to determine the storm tracks: first, by detecting and tracking single cyclones (Gulev et al. 2001; Serreze et al. 1997; Zolina and Gulev 2002) and, second, by high-pass filtering the sea level pressure and geopotential height fields (Blackmon et al. 1984; Cai and Mak 1990; Lau and Nath 1991; Rogers 1990). But with both methods it is not possible to quantify the contribution of the synoptic systems to the lowfrequency variability of the pressure field. This is because high-pass filtering completely separates the frequency bands, and cyclone track algorithms only account for the central pressure, and in addition they do not include high pressure systems on synoptic time scales. In the present study a new filter method is developed. Disturbances of the daily sea level pressure field by synoptic systems are detected at each grid point, and fixed mean values are assigned to each system (cyclone, anticyclone, or neither). The values are averaged over a month to obtain the low-frequency variability of the occurrence of the synoptic systems. Thus, this filtering method does not completely separate the frequency bands as the Fourier filtering does. With this method it is possible to estimate the contribution of the synoptic systems to the low-frequency variability in the pressure field. Primarily we extract the part of the month-to-month variability in the sea level pressure anomalies that is due to the occurrence of the synoptic systems, which is then related to the monthly mean NAO. Additionally we applied the same filtering method to the results of a coupled climate model to understand the influence of the synoptic systems on the decadal variations of the NAO.

A detailed explanation of the filter method and the data used follows in the next section. The results after filtering are discussed for monthly time scales in section 3. In section 4 a physical explanation is given of how the distribution of the synoptic systems can influence the NAO. In section 5 the decadal variations are investigated with the same method. A discussion about the relation of the NAO to the baroclinic zone follows in section 6.

\section{Data and the filtering method}

The filtering method described below is applied to daily sea level pressure anomalies (NCEP-NCAR reanalysis from 1948 to 2001) at each grid point in the North Atlantic region. The anomalies are calculated by removal of the mean annual cycle. To investigate decadal fluctuations as well, we additionally used a 300-yr control integration under present-day conditions of the coupled model ECHAM4/OPYC3 (Roeckner et al. 1996).

The NAO index is calculated from the same data as the difference between normalized monthly mean sea level pressure anomalies near the Azores and Iceland as suggested by Hurrell (1995). The purpose of filtering the sea level pressure anomaly field is to extract the part of the low-frequency variability that depends on the distribution of the synoptic systems. With this lowfrequency variability, we aimed to find out whether the NAO can be explained through the distribution of the synoptic systems alone or whether other low-frequency variations in sea level pressure are involved. As these other low-frequency variations one might consider climatological changes in sea level pressure as for example caused by changes in the position of the ITCZ. The main idea is to separate the low-frequency part of the total time series at each grid point into "synoptic" and "other" components, with the ultimate goal to isolate the synoptic signal.

For the detection of the synoptic systems no common method exists. Cyclone tracking algorithms use different criteria including lifetime, speed, intensity, and deepening rate to detect the central pressure (Gulev et al. 2001; Serreze et al. 1997). Other simpler approaches use thresholds to study the behavior of deep cyclones (Lambert et al. 2002). For our purpose, it is crucial to detect the whole area influenced by the synoptic systems. Additionally, we need to account for cyclones and anticyclones. The latter are rarely characterized in literature. An overview about the filtering method is given in Fig. 1.

The first step of this method is an analysis of the daily pressure anomalies at a given grid point to detect whether a cyclone, anticyclone, or neither exists. If the magnitude of the pressure anomaly is greater than a predetermined, seasonally varying threshold for less than 12 consecutive days, it is labeled "cyclone" or "anticyclone." Thus cyclones and anticyclones have now been flagged in each gridpoint time series.

The second step is to construct a filtered time series from the original data using the flagged systems. If a synoptic system is detected in the time series, all time points associated with that system are assigned a mean 


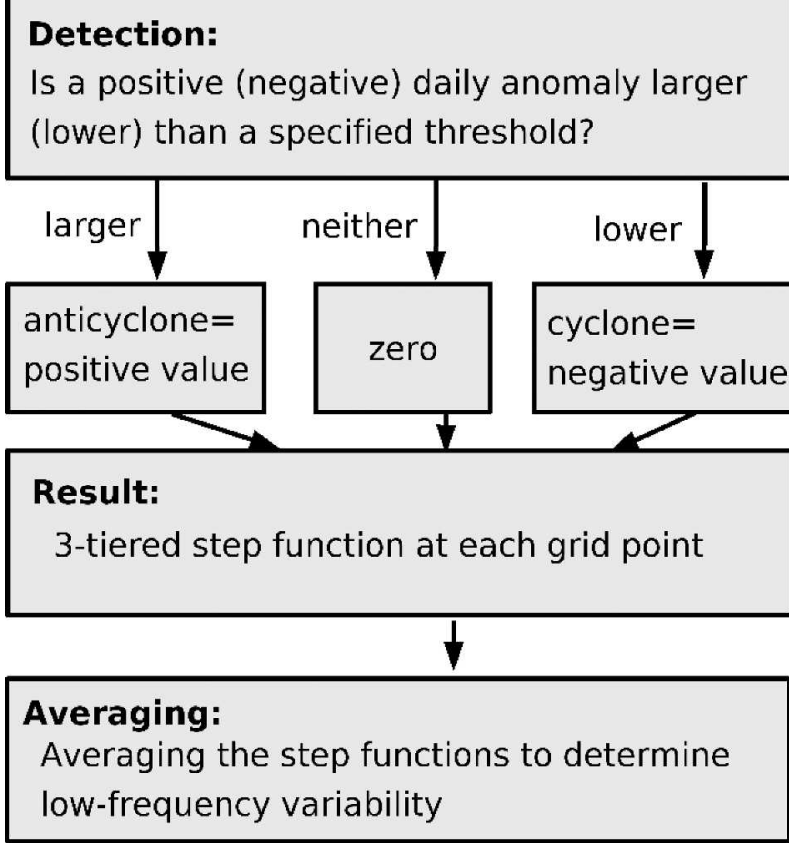

FIG. 1. Scheme of the filtering method.

pressure anomaly (negative/positive for cyclones/ anticyclones) based on its grid point location and time of the year. If no cyclone or anticyclone flag exists in the original time series the filtered time series receives the value zero. Thus the final filtered time series is a three-tiered step function. The filtered time series is then averaged over certain time periods in order to obtain the low-frequency variability that is due to the distribution of the synoptic systems. Local intensity variations in the synoptic systems are excluded by this filtering method. Nevertheless the assigned values vary from grid point to grid point and season to season since these values are determined as mean pressure anomaly of all detected synoptic systems during the whole observing period at each grid point and each season. This is necessary since the anomalies in the sea level pressure field are much larger in the north than in the south. If we would not take this into account, we could not expect to find a realistic NAO pattern in the filtered data.

To illustrate the idea behind the filtering method we generated a set of artificial data: a white noise process is added to a low-frequency sinusoidal process (Fig. 2, left, gray bars). The red curve depicts the 30-day running mean of the entire process, while the blue curve shows the 30-day running mean of the noise process only. Here, the noise process is meant to represent the synoptic-scale fluctuations while the sinusoidal process represents additional low-frequency fluctuations in the pressure field with unknown origin. In the right image of Fig. 2 the filtered time series is shown. The highfrequency fluctuations are detected via thresholds based on the noise process $( \pm 5 \mathrm{hPa})$. The tolerance against the thresholds is high: Low thresholds lead to the detection of many high-frequency events and the magnitude of the assigned value becomes small; with high thresholds on the other hand one would find only a small number of high-frequency fluctuations with a larger magnitude of the assigned values. Both effects compensate in a certain range of thresholds. The blue curve on the right of Fig. 2 shows the 30-day running mean of the filtered process. The time series of the 30-day running means of the noise process and of the filtered time series of the noise plus sinusoidal process
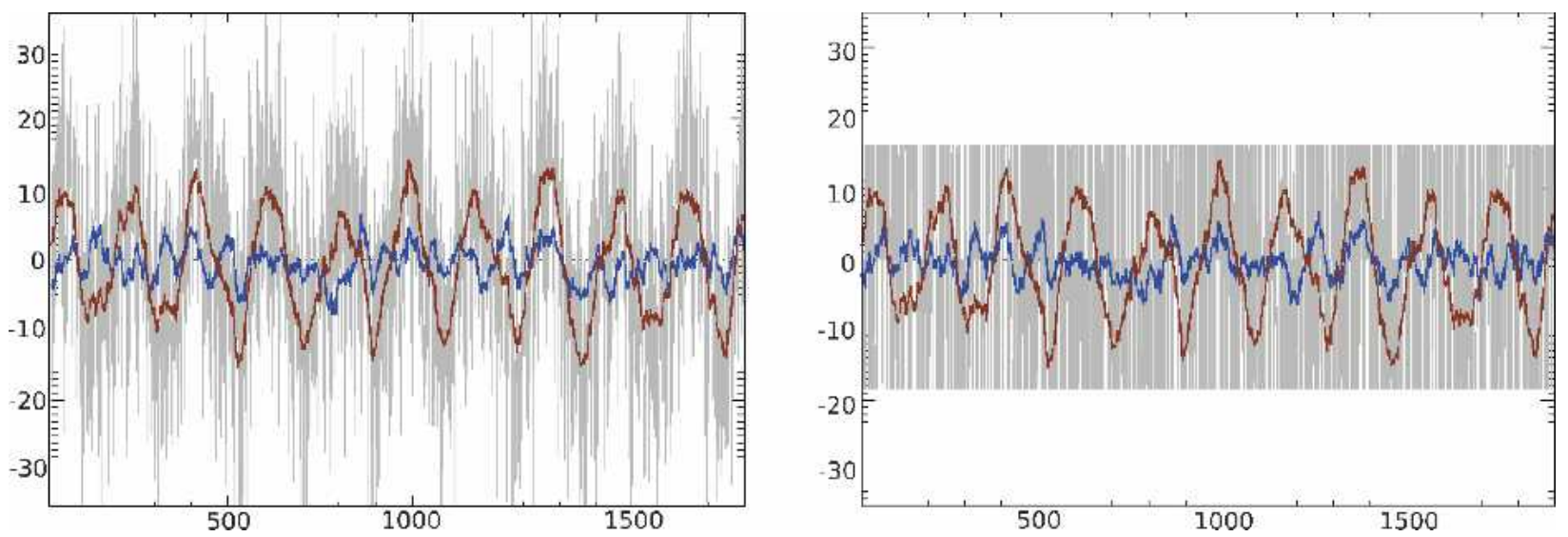

FIG. 2. (left) Time series of a white noise process with a sinusoidal process added to it (gray bars). The red curve depicts the 30-day running mean of the original time series, while the blue curve shows the 30-day running mean of the noise process only. (right) Time series of the above process but filtered. The blue curve shows the 30-day running mean of the filtered process, and the red curve shows the 30-day running mean of the original time series. 
agree almost perfectly. This fact demonstrates that with the presented method we extract in this example the portion of the low-frequency variability, which is caused by the high-frequency fluctuations. The lowfrequency variation produced by the sinusoidal process is eliminated. Regarding sea level pressure anomalies, we identified the high-frequency variability with the synoptic systems. Thus, our method enables us to estimate the contribution of the synoptic systems, mainly due to the distribution of the synoptic systems, to the low-frequency variability in the pressure field. In case that the original low-frequency variability would differ greatly from the low-frequency variability of the filtered data, other sources of low-frequency variability have to be considered, which are not caused by the distribution of the synoptic systems.

The detection of the synoptic systems is based on the assumption that a cyclone (anticyclone) lowers (raises) the surface pressure for a certain time when it passes a certain location. This time period of influence is selected as 12 days, according to the synoptic time scale. If the magnitude of the pressure anomaly is greater than a predetermined, seasonally varying threshold for 12 or less consecutive days, it is labeled "cyclone" or "anticyclone," respectively. The predetermined seasonally varying thresholds for cyclones $\left(S_{Z}\right)$ and anticyclones $\left(S_{A}\right)$ are as follows: $S_{Z}=-11$ and $S_{A}=6 \mathrm{hPa}$ in winter; $S_{Z}=-8$ and $S_{A}=5 \mathrm{hPa}$ in spring and autumn; and $S_{Z}=-6$ and $S_{A}=4 \mathrm{hPa}$ in summer. The determination of the thresholds is problematic by nature. Too small values lead to problems with the time limit of 12 days, for example, in the case of fast cyclones overrunning stationary lows, while too extreme values hinder the detection of weak synoptic systems.

A preliminary determination of the thresholds were carried out by subjective inspection of the pressure field (distribution of cyclones and anticyclones). In a second, more quantitative step we calculated the product of the mean intensity of the systems and their time duration. Those thresholds are selected that optimize this product. With this product, we expect to yield the highest contribution of the synoptic systems to the lowfrequency variability of the sea level pressure. We missed, however, extreme synoptic systems. Therefore, we did a second run through the data with more intense thresholds $\left(S_{Z}^{\prime}=S_{Z}-7 \mathrm{hPa}\right.$ and $\left.S_{A}^{\prime}=S_{A}+8 \mathrm{hPa}\right)$, which identifies most of the missed systems. The final results demonstrate that the sensitivity of the filtering against small changes $(1-3 \mathrm{hPa})$ in the thresholds is relatively weak.

To check whether this method really works well we carried out different tests. A supporting detection method is applied utilizing deviations from the zonal mean. The thresholds to detect the systems via deviation from the zonal mean are latitude dependent, with $\tilde{S}_{Z}=-8 \mathrm{hPa}$ and $\tilde{S}_{A}=+8 \mathrm{hPa}$ in winter and $\tilde{S}_{Z}=-6$ $\mathrm{hPa}$ and $\tilde{S}_{A}=+6 \mathrm{hPa}$ in summer around Iceland. In the vicinity of the Azores the absolute values of the thresholds are about $1-2 \mathrm{hPa}$ less. This method is relatively robust concerning the thresholds, too. A problem arises at the high latitudes. The results are unrealistic north of $70^{\circ} \mathrm{N}$ and already north from $65^{\circ} \mathrm{N}$ there is a tendency for inaccuracies. However, in the midlatitudes both methods lead to very similar results. At all midlatitude grid points the time series that result from the two filtering methods agree well, with significant correlations of more than 0.8 . Thus, in the midlatitudes the choice of the method to detect the synoptic systems does not crucially influence the results.

In a second test the synoptic disturbances passing Iceland and the Azores during winter 1970/71 are manually determined from the analyzed sea level pressure charts. The time series of this analysis compares well with the results of the filter method. In particular, the low-frequency variability of the two time series are in good agreement.

To demonstrate that the majority of the synoptic systems are detected, in Fig. 3 Hovmoeller diagrams are shown of the sea level pressure anomalies averaged from $55^{\circ}$ to $65^{\circ} \mathrm{N}$ for the winters 1969 and 1989. (Light gray regions represent positive values of more than 10 $\mathrm{hPa}$, and dark gray regions represent negative values less than $-15 \mathrm{hPa}$ ). For both winters, the main features of the original and filtered data agree well. The year 1969 had a pronounced low NAO index and 1989 had a particularly high index. So it is not surprising that in 1989 many more cyclones are observed in the region around Iceland than in 1969. This dependence between the number of cyclones in the North Atlantic region per winter and the NAO index was already shown in other studies (Ubl 2001). The open question remains to which degree the synoptic systems are responsible for the NAO pattern.

\section{Variations in sea level pressure on monthly time scale}

Because of the common definition of the NAO, knowledge about the causes of the pressure variability at the two locations of Iceland and the Azores is important for better understanding of the NAO. Thus, the influence of the distribution of the synoptic systems on the long periodic sea level pressure fluctuations $(>30$ days) is investigated at the two grid points near Iceland and the Azores. In Fig. 4 time series are shown of sea level pressure anomalies near Iceland (left) and the 

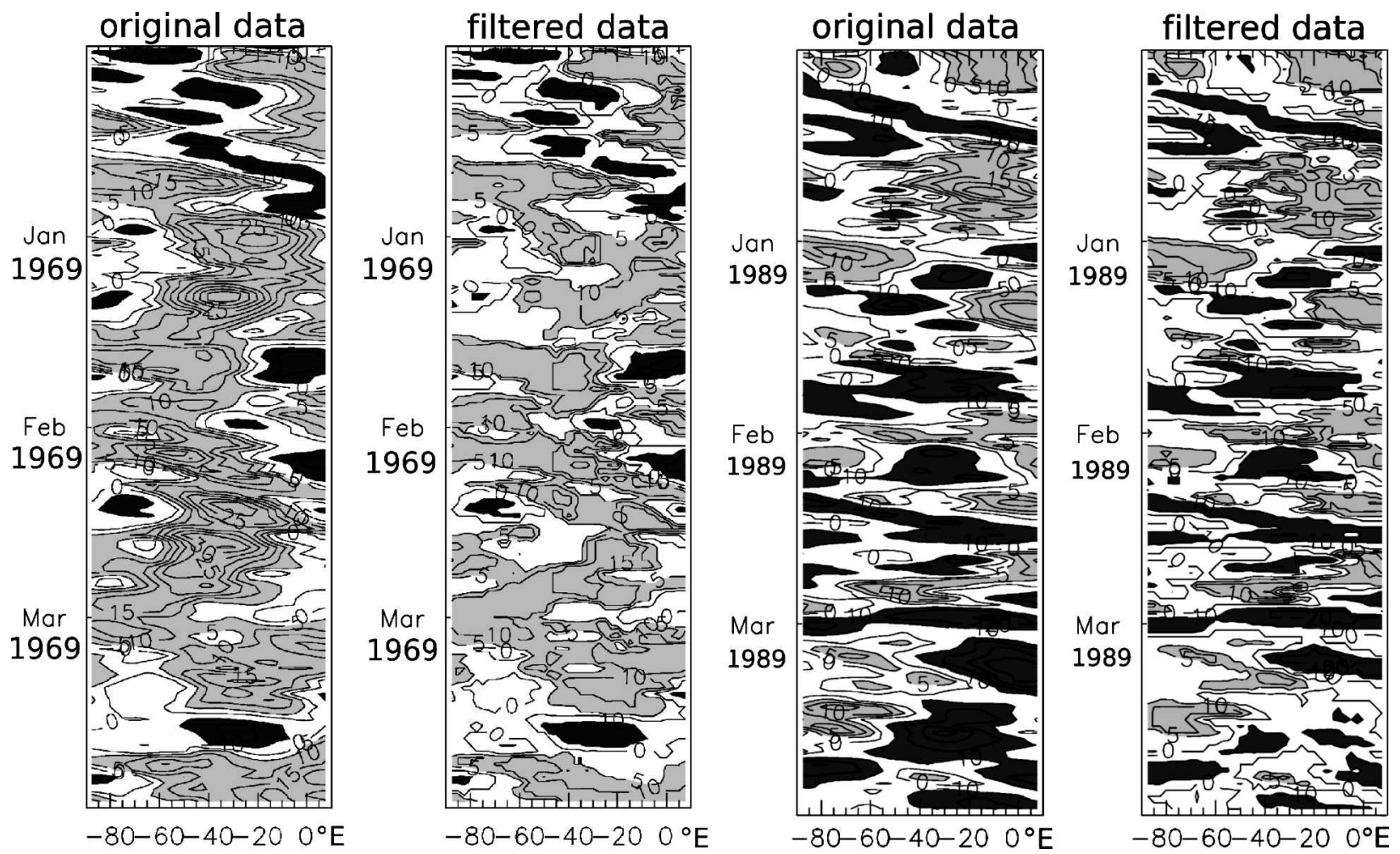

FIG. 3. Hovmoeller diagrams of sea level pressure anomalies for the winters (left) 1969 and (right) 1989 of the original and filtered data, averaged from $55^{\circ}$ to $65^{\circ} \mathrm{N}$. The original data are in both cases shown to the left. Light gray regions represent positive values of more than $10 \mathrm{hPa}$, and dark gray regions negative values less than $-15 \mathrm{hPa}$.

Azores (right). [The light gray bars represent the original, and the darker gray bars the filtered, data. The colored lines depict the 30-day running means of the original (blue) and the filtered data (red)]. The agreement between these two running means is very high [correlations of 0.96 (Iceland) and 0.94 (Azores)]. Only

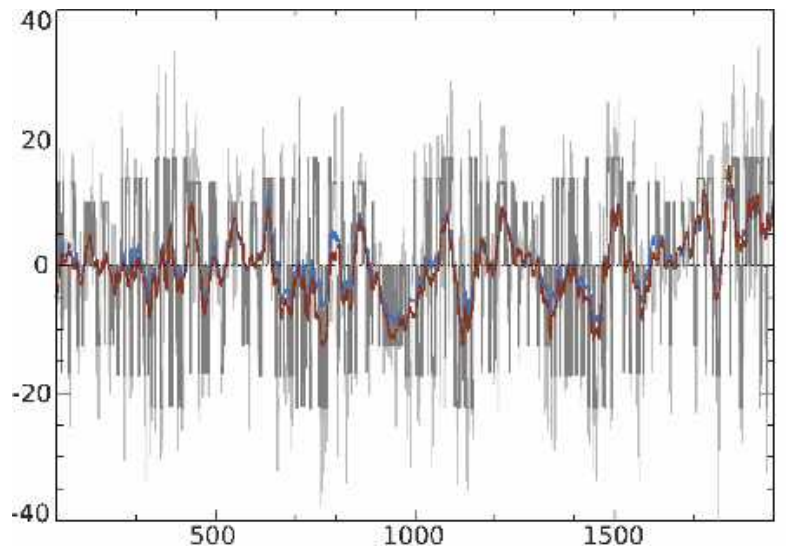

the extreme values are too weak in the filtered data. The differences may have two causes: first, they are results of not-detected synoptic systems or small contribution by underestimation of extraordinarily intense systems; second, a small contribution by other than synoptic variability. In any case, the bulk of anomalous

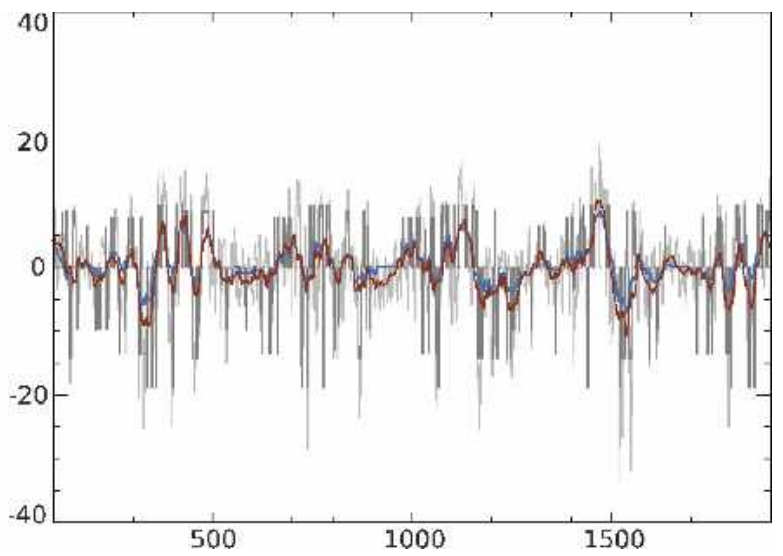

FIG. 4. A 5-yr section of the daily sea level pressure anomalies (hPa) near (right) the Azores and near (left) Iceland (gray) compared with the corresponding filtered data (dark gray). Thick lines depict the 30-day running means of original (red) and filtered data (blue). The correlation between the 30-day running means of the original and filtered total time series (1948-2001) is 0.96 for Iceland and 0.94 for the Azores. 

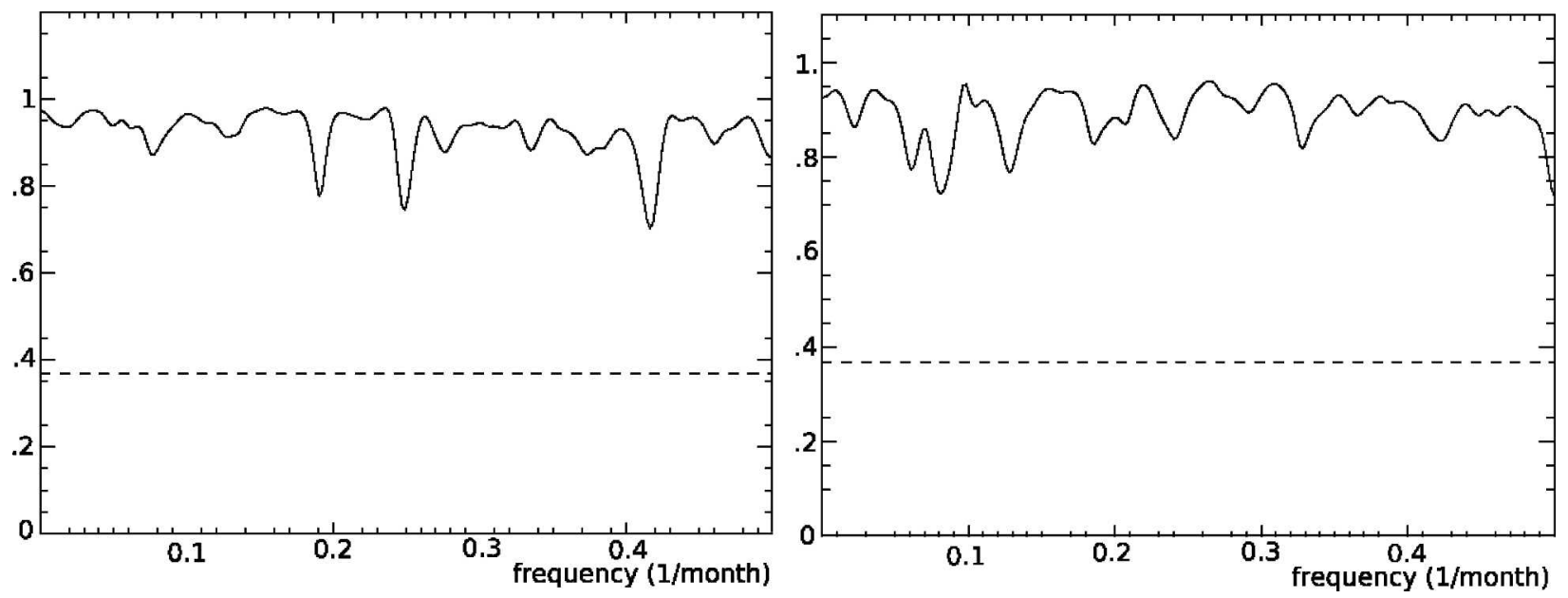

FIG. 5. Squared coherency between monthly filtered and original sea level pressure on (left) Iceland and (right) the Azores

(NCEP-NCAR). The dashed line marks the $95 \%$ significance level.

fluctuations in the sea level pressure near Iceland and the Azores is obviously due to the distribution of the occurrence of the synoptic systems, and there is very little room for additional causes of low-frequency variations in the sea level pressure field. This result agrees well with that of Madden (1976): "we would conclude, that in middle latitudes there is little evidence in the dataset (here sea level pressure) that there exists any low-frequency variability over and above an unpredictable low-frequency white noise extension of daily weather fluctuations." We can additionally explain our results: the interannual oscillation in the analyzed sea level pressure field is dominated by the variations of the number and distribution of synoptic systems.

Since we eliminate with our filter method intensity variations of the synoptic systems, the intensity variations of the synoptic systems have little effect on the low-frequency variability of the surface pressure over the North Atlantic. To check whether this is true for all time scales, cross spectra are calculated between the monthly averaged original and filtered time series (Fig. $5)$. The relation between the two time series is high in general. In all frequency ranges the squared coherency is greater than 0.8 , indicating that the agreement is large at all time scales. Thus, the low-frequency variations of the sea level pressure at the two reference points of the NAO are hardly dependent on intensity changes of synoptic systems or other low-frequency variations, but depend above all on the distribution of the synoptic systems over the North Atlantic.

\section{The coupling of the centers of the NAO}

To explain the effect of the distribution of the synoptic systems on the NAO, we assume a mean large- scale pressure gradient between Iceland and the Azores, which is disturbed by the synoptic systems. From synoptic observations (inspection of weather charts over the North Atlantic) and cyclone tracking (Gulev et al. 2001) it is well known that cyclones rarely track parallel across the North Atlantic. In general one observes at a certain time and a certain longitude only one cyclone (Hoskins and Valdes 1990). If one cyclone is found in the north near Iceland, pressure decreases there and since no other cyclone is observed in the south of it at the same time, pressure remains relatively high near the Azores. Analogously, a southern cyclone track causes a decrease of the pressure in the southern North Atlantic while the pressure remains constant in the northern part. Because of this mechanism in combination with a certain persistence of the cyclone tracks, a coupling occurs between the northern and southern parts of the North Atlantic even on longer time scales. In addition, the mean cyclone pressure anomalies are lower, which means the variance is greater, in the northeast North Atlantic than in the south (Fig. 6), which makes this region in an EOFanalysis more dominant than other regions.

According to these considerations it makes sense that the first EOF of sea level pressure anomalies over the North Atlantic shows a dipole-like structure even on time scales much larger than those of cyclones. The first and second EOFs and principal components (PCs) of the 30-day running mean of the original sea level pressure anomalies in the North Atlantic region are depicted in Fig. 7. The first EOF shows clearly the NAO pattern, while the second EOF is dominated by a single pole in the central east Atlantic (east Atlantic pattern). Figure 8 shows the first and second EOF of the filtered 


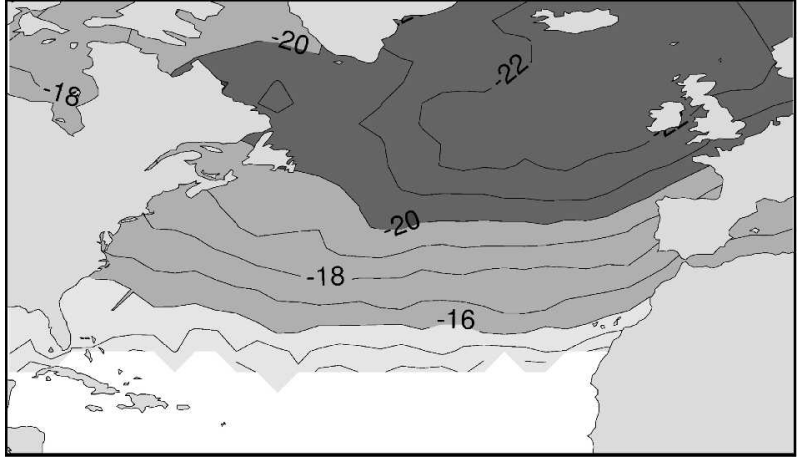

FIG. 6. Mean pressure anomalies of the detected cyclones (hPa) for the North Atlantic region of the winters (DJF) 1948-2001.

data together with the corresponding PCs. For comparison, the PCs of the original sea level pressure anomalies are shown as well (gray). The patterns of these EOFs agree well with those of Fig. 7. Correlations between the PCs are almost perfect and the explained variances of the EOFs are very similar to those of the original data. The amplitudes of the filtered data are too weak at about $2 \mathrm{hPa}$. This may be partly due to difficulties already discussed in section 3 . To quantify the effect of missing synoptic systems, we give an example: if one misses a cyclone in the north influencing one grid point for 3 days, this leads to an error of approximately $2 \mathrm{hPa}$ during a period of 30 days. Additionally the exclusion of the intensity variations in the synoptic systems causes an error of the order of $\pm 1 / 2$ $\mathrm{hPa}$ (standard deviation of the 30-day running means when varying the intensities of the synoptic systems in the observed range). But altogether, we can conclude from these results that the distribution of the synoptic systems alone is sufficient to produce the NAO centers in the EOF analysis.

\section{Decadal variability}

To investigate the role of the distribution of the synoptic systems on the NAO on decadal time scales, we used the results of a 300-yr control integration under present-day conditions of the global coupled general circulation model ECHAM4/OPYC3. The atmospheric component is the fourth generation of a hierarchy of models that was further developed at the Max-PlanckInstitut für Meteorologie (MPI) in Hamburg, Germany, from the former European Centre for Medium-Range Weather Forecasts (ECMWF) model. It has a horizontal resolution of T42 and 19 levels in the vertical (Roeckner et al. 1996). OPYC3 is a three-component ocean model including an interior ocean, a mixed-layer component, and a sea ice model (Oberhuber 1993). The daily sea level pressure anomalies are filtered as described in section 2. Figure 9 shows the 100-month running means of the original sea level pressure anomalies and the filtered data near Iceland and the Azores. The result is very similar to the investigation on monthly
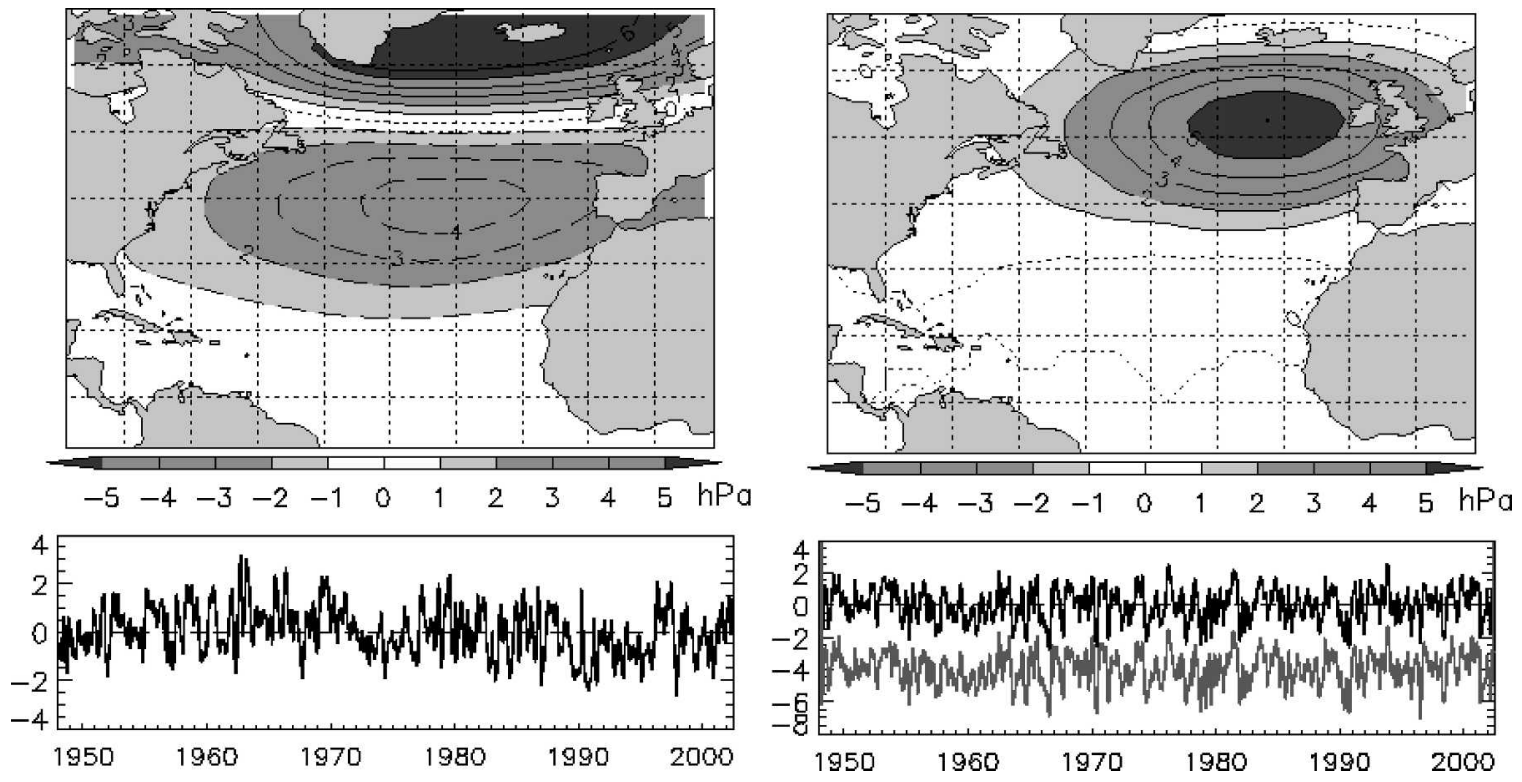

FIG. 7. (left) First and (right) second EOF and corresponding PCs of the 30-day running mean of the sea level pressure anomalies in the North Atlantic region. Explained variance: 37\% (EOF1) and 24\% (EOF2). Full lines mark positive, and dashed lines negative, values. 


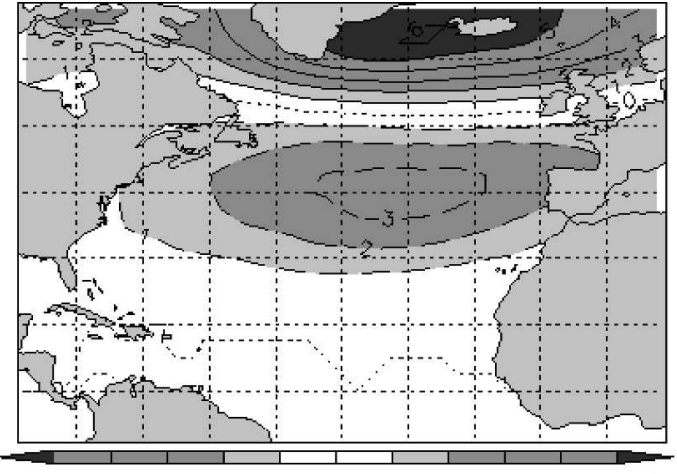

$\begin{array}{lllllllllllll}-5 & -4 & -3 & -2 & -1 & 0 & 1 & 2 & 3 & 4 & 5 & \mathrm{hPO}\end{array}$

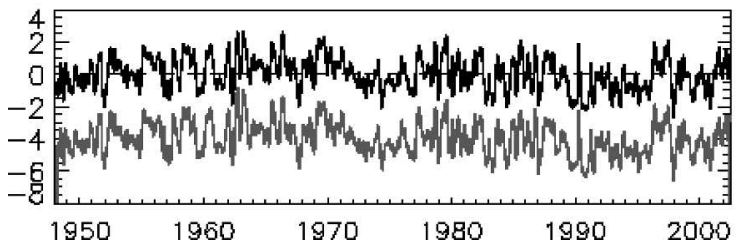

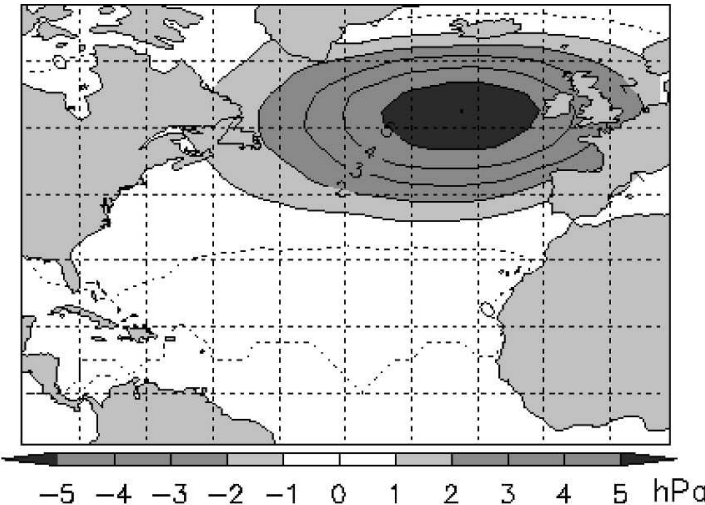

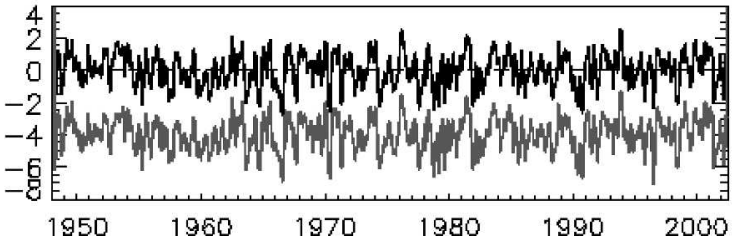

FIG. 8. (left) First and (right) second EOF and PCs of the 30-day running mean of the filtered data; in addition first and second PCs of observed sea level pressure anomalies are given for comparison with displaced mean value (gray). The portions of explained variances are $35 \%$ (EOF1) and 25\% (EOF2); correlations between the PCs of the original and the filtered data are 0.99 (PC1) and 0.98 (PC2). Full lines mark positive, and dashed lines negative, values.

time scales with the NCEP-NCAR data. The agreement between the filtered and the original data is high [correlations of 0.92 (Iceland) and 0.91 (Azores)]. That means for decadal time scale, the evolution in time can almost completely be explained through the distribution of the synoptic systems. The same is true for the spatial pattern. The EOFs of 100-month running means of the original data agree well with those of the filtered data (Fig. 10). The correlation of the PCs is 0.97. Even though the pattern for the filtered data is slightly too weak, which might be again partly due to undetected synoptic systems or a small contribution by other varia- tions, the bulk of the NAO pattern can be explained through the distribution of the synoptic systems.

\section{Discussion}

The distribution of the synoptic systems over the North Atlantic in space and time mainly depends on the total number of generated systems in their source region and the tracks they take across the North Atlantic. The number of systems that cross the North Atlantic varies considerably from year to year. The total number of cyclones lies between approximately $50-100$ cyclones
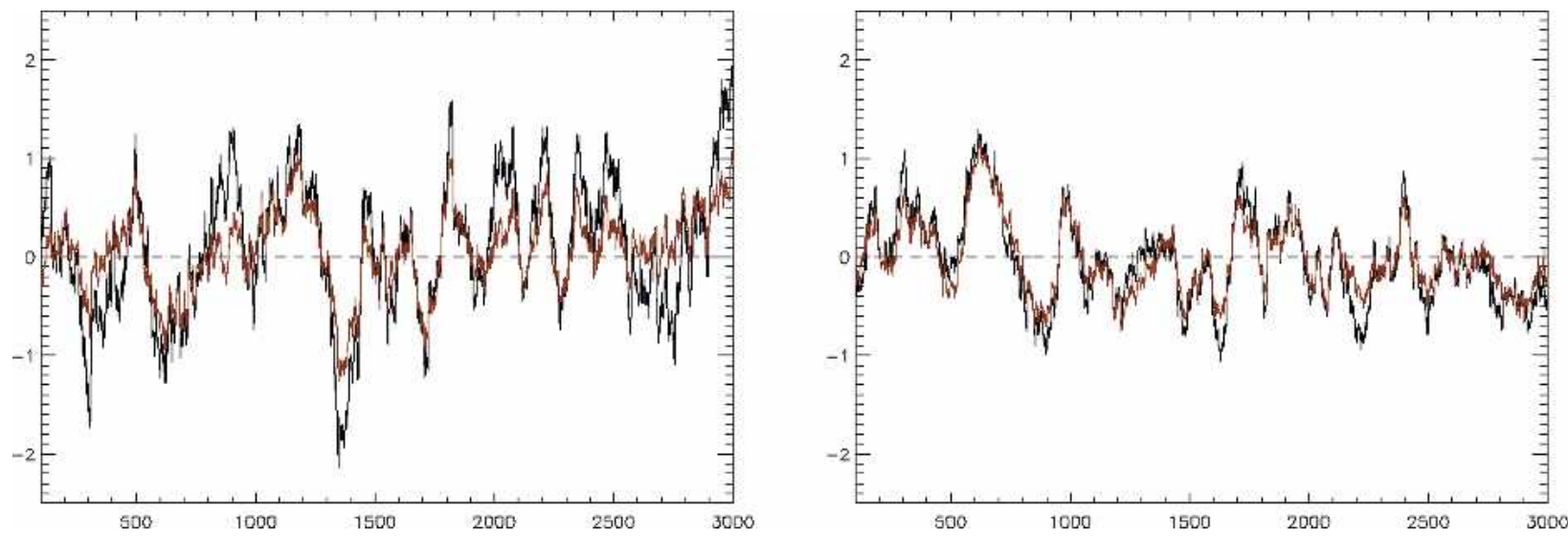

FIG. 9. Time series of the 100-month running means of the original sea level pressure anomalies (black) and the filtered data (red) near (left) Iceland and (right) the Azores. 

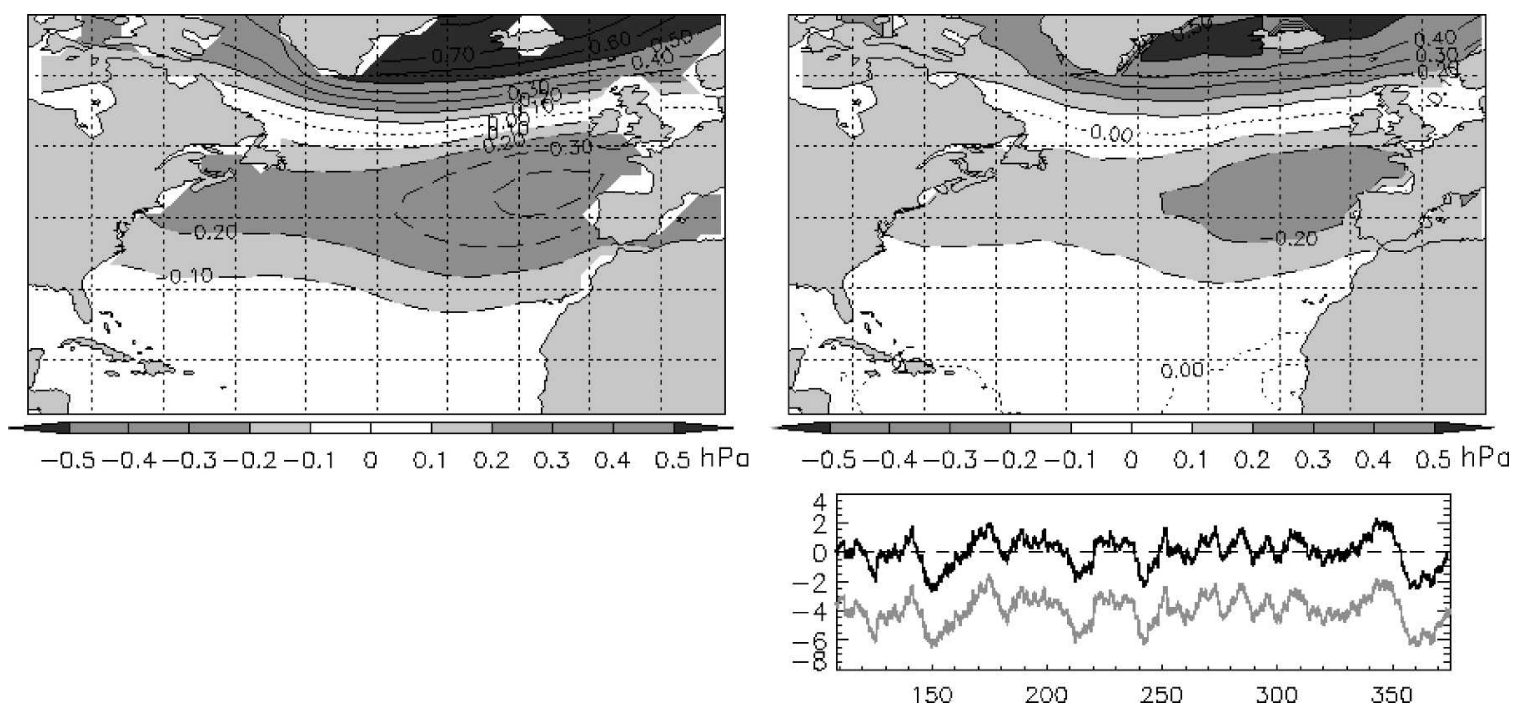

FIG. 10. First EOF of the 100-month running mean of (left) sea level pressure anomalies (explained variance: $38 \%$ ) and (right) the filtered data $(37 \%)$. The corresponding PCs are shown below the right figure. The PC of the original data is displaced (gray); the correlation of the PCs is 0.97. Full lines mark positive, and dashed lines negative, values.

per winter season [December-January-FebruaryMarch (DJFM)]; this high variability is superimposed by a negative trend (Gulev et al. 2001). In connection with the NAO, one would rather expect a positive trend according to a positive NAO during the last decades. But, a comparison of the total number of cyclones crossing the North Atlantic with the NAO index does not show high correspondence (Gulev et al. 2001). Thus, we hypothesize that variations of the storm tracks are the main source of variability of the NAO index, not the total number of cyclones. Numerous studies have documented a relationship between storm tracks or tracks of synoptic baroclinic waves and long planetary waves or the extratropical jet streams (Blackmon et al. 1984; Cai and Van den Dool 1991; Cai and Mak 1990; Lau 1988; Lau and Nath 1991; Thompson et al.
2003). The synoptic waves need baroclinic unstable large-scale zonal flow to be generated. But they can feed back to strengthen the planetary waves or the jet streams. Over the North Atlantic typically a stationary, slowly varying trough exists off the east coast of the American continent. The baroclinic zone of this trough extends toward the northeast. It can be described by the position of the temperature gradient in the middle troposphere-baroclinic front. We analyzed the mean winter position of the front at the 400-hPa level. We define the position of the front as the region with the highest temperature gradient between the $-28^{\circ}$ and $-42^{\circ} \mathrm{C}$ isotherms at the $400-\mathrm{hPa}$ level in the questionable range of latitude. Figure 11 shows the mean winter (DJFM) temperature at $400 \mathrm{hPa}$ and the position of the front between $90^{\circ} \mathrm{W}$ and $10^{\circ} \mathrm{E}$ for a high (1989) and a low NAO
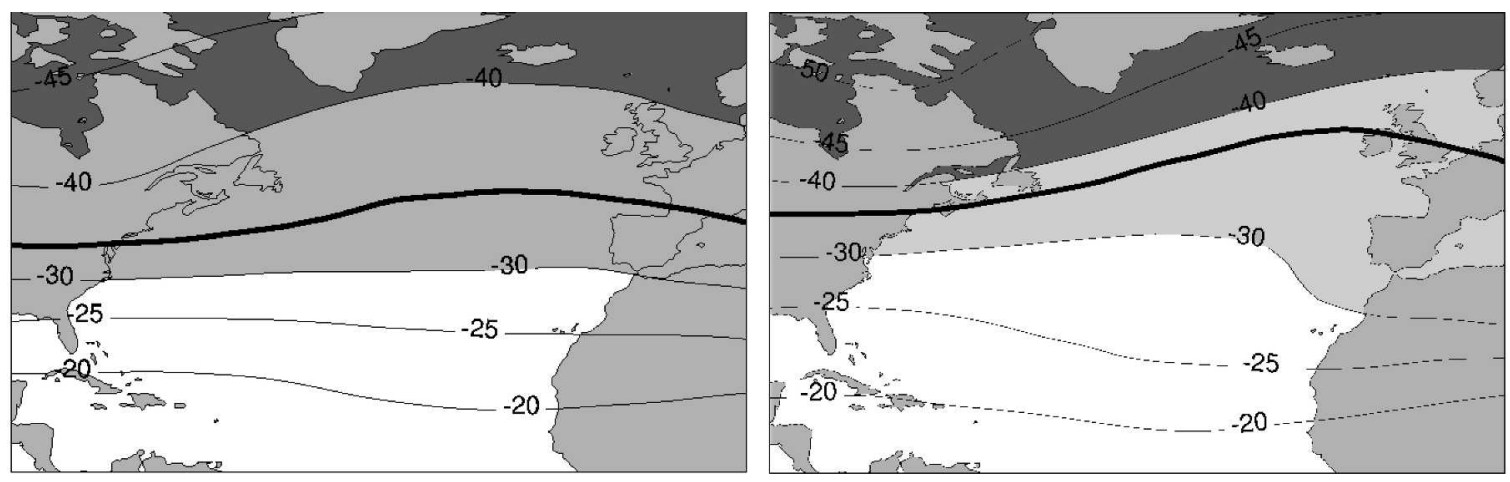

FIG. 11. Seasonal-averaged (DJFM) 400-hPa temperature in (left) 1969 (low NAO) and (right) 1989 (high NAO); the full line depicts the mean position of the polar front during winter. 


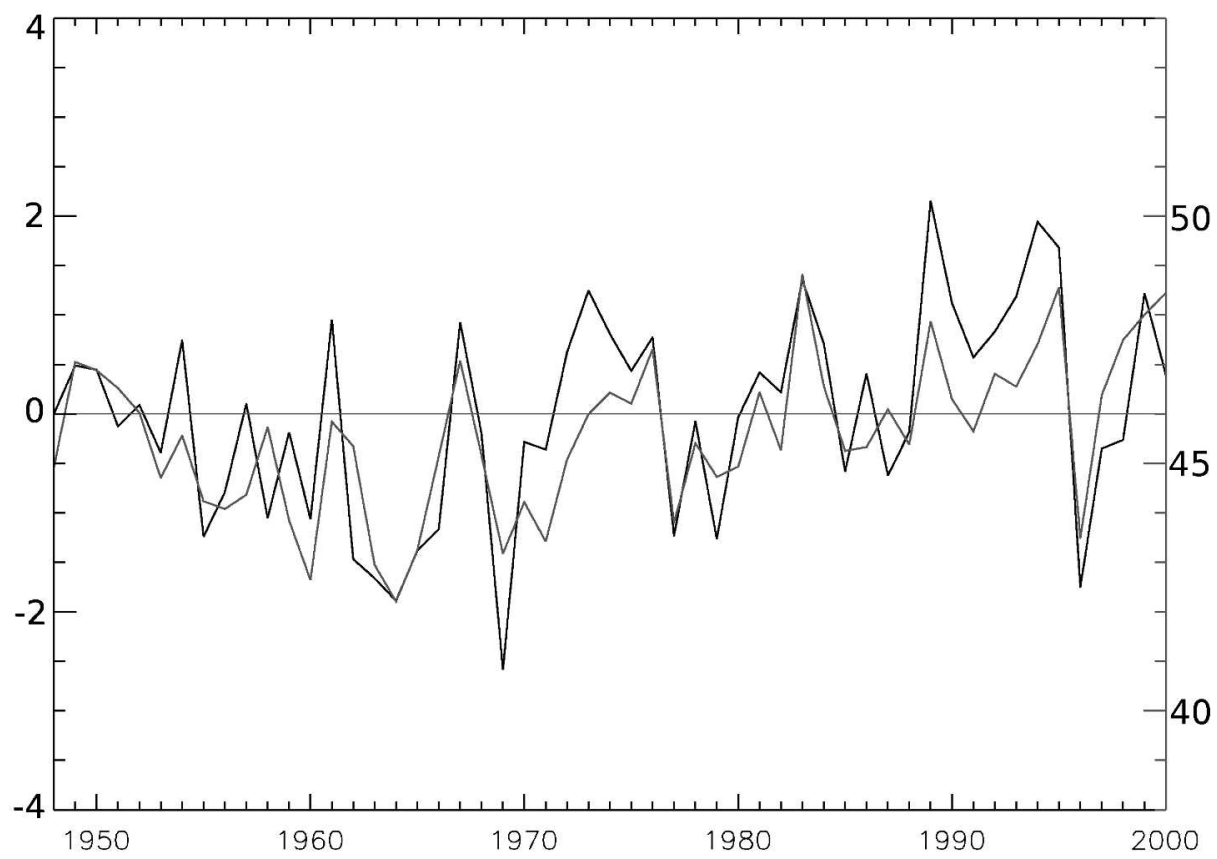

FIG. 12. Winter NAO index (black) and the mean winter position of the polar front (light gray) between $90^{\circ} \mathrm{W}$ and $10^{\circ} \mathrm{E}$ in $400 \mathrm{hPa}(\mathrm{DJFM}$ ); the correlation is 0.78 (the $95 \%$ significance level is $0.28)$.

year (1969). In the year with a high NAO index the front is obviously located much more to the north, and in addition, the baroclinity over the western and central Atlantic is much greater than in the year with low NAO index.

To investigate this relationship closer, we depict the time series of the mean position of the front during the winter seasons and of the NAO index at Fig. 12. The NAO index follows the position of the polar front closely. The significant correlation coefficient is 0.78 (the $95 \%$ significance level is 0.28 ). This fact supports our hypothesis that the intensity of the NAO depends mainly on the position of the cyclone tracks that correspond to the position of the polar front (polar jet) in the middle troposphere, here at $400 \mathrm{hPa}$.

\section{Conclusions}

The present study shows that the distribution of the synoptic systems is mainly responsible for lowfrequency variability of the sea level pressure field over the North Atlantic and is sufficient to generate the NAO pattern. This holds for monthly NCEP-NCAR data as well as for decadal fluctuations in the global coupled model ECHAM4/OPYC3. Intensity variations in the synoptic systems do not play an important role, except through displacement of the tracks. Synoptic systems with northern tracks are of greater intensity than those of southern tracks. But local variations in the intensity are not of importance for the low-frequency variability of sea level pressure. The position of the cyclone tracks mainly determines the strength of the NAO. Since cyclones need a baroclinic instability for their generation and further development it follows that the NAO index is well correlated with the latitudinal position of the polar front. Because of the relationship between the storm tracks and the large-scale trough over the North Atlantic - the position of which is dynamically related to the condition over the North American continent-the variations of the NAO are well related to the conditions over the North American continent and its contrast with the North Atlantic waters.

Acknowledgments. This work is a contribution to the Sonderforschungsbereich 460, "Dynamics of Thermohaline Circulation Variability," at the University of Kiel supported by the German Research Foundation. Thanks also go to Dr. K. Lohmann from the MPI in Hamburg for providing the GCM data.

\section{REFERENCES}

Benedict, J. J., S. Lee, and S. B. Feldstein, 2004: Synoptic view of the North Atlantic Oscillation. J. Atmos. Sci., 61, 121-144. 
Blackmon, M. L., Y. H. Lee, and L. M. Wallace, 1984: Horizontal structure of $500 \mathrm{mb}$ height fluctuations with long, intermediate and short time scales. J. Atmos. Sci., 41, 961-979.

Cai, M., and M. Mak, 1990: Symbolic relation between planetary and synoptic-scale waves. J. Atmos. Sci., 47, 2953-2968.

_- and H. M. Van den Dool, 1991: Low-frequency waves and traveling storm tracks. Part I: Barotropic component. J. Atmos. Sci., 48, 1420-1436.

Gulev, S. K., O. Zolina, and S. Grigoriev, 2001: Extratropical cyclone variability in the Northern Hemisphere winter from the NCEP/NCAR reanalysis data. Climate Dyn., 17, 795-809.

Hoskins, B. I., and P. J. Valdes, 1990: On the existence of storm tracks. J. Atmos. Sci., 47, 1854-1864.

Hurrell, J. W., 1995: Decadal trends in the North Atlantic Oscillation: Regional temperatures and precipitation. Science, 269, 676-679.

Jones, P. D., T. Jonsson, and D. Wheeler, 1997: Extension to the North Atlantic Oscillation using early instrumental pressure observations from Gibraltar and south-west Iceland. Int. J. Climatol., 17, 1433-1450.

Lambert, S. J., J. Sheng, and J. Boye, 2002: Winter cyclone frequencies in thirteen models participating in the Atmospheric Model Intercomparison Project (AMIP1). Climate Dyn., 19, $1-16$.

Lau, N. C., 1988: Variability of the observed midlatitude storm tracks in relation to low-frequency changes in the circulation pattern. J. Atmos. Sci., 45, 2718-2743.

— tropic transient eddy forcing associated with monthly changes in the mid latitude storm tracks. J. Atmos. Sci., 48, 2589-2613.

Madden, R., 1976: Estimates of natural variability of timeaveraged sea-level pressure. Mon. Wea. Rev., 104, 942-952.

Oberhuber, J. M., 1993: The OPYC ocean general circulation model. Tech. Rep. 7, Deutsches Klimarechenzentrum GmbH, Hamburg, Germany, 172 pp.

Roeckner, E., J. M. Oberhuber, A. Bacher, M. Christoph, and
I. P. Kirchner, 1996: ENSO variability and atmospheric response in a global coupled atmosphere-ocean GCM. Climate Dyn., 12, 737-754.

Rogers, J. C., 1990: Patterns of low-frequency monthly sea level pressure variability (1899-1986) and associated wave cyclone frequencies. J. Climate, 3, 1364-1379.

Ruprecht, E., S. Schroeder, and S. Ubl, 2002: On the relationship between the NAO and water vapour transport towards Europe. Meteor. Z., 11, 395-401.

Serreze, M., F. Carse, R. Barry, and J. Rogers, 1997: Icelandic low cyclone activity: Climatological features, linkages with the NAO, and relationships with recent changes in the Northern Hemisphere circulation. J. Climate, 10, 453-464.

Thompson, D. W. J., and J. M. Wallace, 1998: The Arctic Oscillation signature in the wintertime geopotential height and temperature fields. Geophys. Res. Lett., 25, 1297-1300.

- and - 2000: Annular modes in the extratropical circulation. Part I: Month-to-month variability. J. Climate, 13, 10001016.

- S. Lee, and M. P. Baldwin, 2003: Atmospheric processes governing the North Hemisphere annular mode/North Atlantic Oscillation. The North Atlantic Oscillation-Climate Significance and Environmental Impact, Geophys. Monograph, No. 134, Amer. Geophys. Union, 81-112.

Ubl, S., 2001: Variabilität von Zykloneneigenschaften über dem Nordatlantik. M.S. thesis, University of Kiel, Kiel, Germany, $68 \mathrm{pp}$.

Walker, G. T., 1924: Correlation in seasonal variation of weather, IX. Mem. Indian Meteor. Dept., 24 (9), 275-332.

Wanner, H., S. Broennimann, C. Casty, D. Gyalistras, J. Lutterbacher, C. Schmutz, D. Stephenson, and E. Xoplaki, 2001: North Atlantic Oscillation-Concepts and studies. Surv. Geophys., 22, 321-382.

Zolina, O., and S. K. Gulev, 2002: Improving the accuracy of mapping cyclone numbers and frequencies. Mon. Wea. Rev., 130, 748-759. 